\title{
Global Research Highlights
}

Editor's note: CJEM has partnered with a small group of selected journals of international emergency medicine societies to share from each a highlighted research study, as selected monthly by their editors. Our goals are to increase awareness of our readership to research developments in the international emergency medicine literature, promote collaboration among the selected international emergency medicine journals, and support the improvement of emergency medicine world-wide, as described in the WAME statement at http://www.wame.org/about/policy-statements\#Promoting\%20Global\%20Health. Abstracts are reproduced as published in the respective participating journals and are not peer reviewed or edited by CJEM.

\section{Annals of Emergency Medicine}

www.acep.org/annals/

Official journal of the American College of Emergency Physicians (The print version of this article has been scheduled for November 2018)

\section{The Characteristics and Outcomes of Patients Who Make an Emergency Department Visit for} Hypertension After Use of a Home or Pharmacy Blood Pressure Device

Clare L. Atzema, Alyssa Wong, Sameer Masood, Ayesha Zia, Suad Al-bulushi, Qazi Z. Sohail, AhmedCherry, Florence S. Chan

Study objective: Emergency department (ED) visits for hypertension are rapidly increasing. Hypertension guidelines began recommending use of self-measurement blood pressure (BP) devices in the early 2000s, which could be contributing to the increase. We aim to examine the proportion of ED visits for hypertension that occurred after a BP measurement with a self-measuring device, and the associated outcomes.

Methods: This retrospective observational study included adults who made an ED visit and received a primary diagnosis of hypertension at 1 of 5 community and tertiary hospitals in Ontario, Canada, between April 2010 and March 2011. We categorized ED visits into 4 groups: those made after a home $\mathrm{BP}$ reading, a pharmacy reading, as a result of a physician referral, and all others (termed "not documented"). We examined rates of admission to the hospital, ED treatment (administration of antihypertensive medication and prescription provision), and post-ED clinical events (follow-up care, return ED visits, and long-term all-cause mortality) by group. Results: Among 1,508 qualifying patients, median presenting ED BP was 182/97 mm Hg (interquartile range 164 to 200/85 to $109 \mathrm{~mm} \mathrm{Hg}$ ). The percentage of patients who presented to the ED after a reading at home, at a pharmacy, or by physician referral and the remaining patients not documented was 40.9\%, 8.3\%, 13.3\%, and 37.5\%, respectively. Overall, 109 patients $(7.2 \%)$ were admitted to the hospital. The proportion of patients admitted after a home or pharmacy reading, after a physician referral, or who were not documented was $3.1 \%$, $11.9 \%$, and $11.0 \%$, respectively. Two-year mortality rates were $5.4 \%, 0.0 \%, 6.5 \%$, and $5.3 \%$ for the home, pharmacy, physician referral, and not documented groups, respectively. Among the $92.8 \%$ of patients who were discharged from the ED, $11 \%$ made another ED visit for hypertension within a year.

Conclusion: In this study, half of patients with an ED visit that was primarily for hypertension presented after elevated readings on self-measurement devices. Only $3 \%$ of these patients were admitted to the hospital. How to cite this article: Atzema, CL, Wong, A, Masood, S, et al. The Characteristics and Outcomes of Patients Who Make an Emergency Department Visit for Hypertension After Use of a Home or Pharmacy Blood Pressure Device. 2018. https://doi.org/10. 1016/j.annemergmed.2018.06.002 


\section{African journal of emergency medicine}

afjem.com

The official journal of the African Federation for Emergency Medicine, the Emergency Medicine Association of Tanzania, the Emergency Medicine Society of South Africa, the Egyptian Society of Emergency Medicine, the Libyan Emergency Medicine Association, the Ethiopian Society of Emergency Medicine Professionals, the Sudanese Emergency Medicine Society, the Society of Emergency Medicine Practitioners of Nigeria and the Rwanda Emergency Care Association

\section{A cross-sectional survey of child abuse management knowledge among emergency medicine personnel in Cape Town, South Africa}

Dessena B, Mullan PC

Afr J Emerg Med 2018; 8(2):59-63

https://doi.org/10.1016/j.afjem.2018.01.005

Introduction: Child abuse is a common condition in the emergency centres of South Africa. It is critical for both prehospital emergency care practitioners and emergency centrebased emergency medicine registrars to be competent in screening, diagnosing, treating, and documenting child abuse. Our goal was to assess the knowledge of child abuse management in a sample of prehospital emergency care practitioners and emergency medicine registrars in Cape Town, South Africa.

Methods: A mixed-methods approach of quantitative and qualitative data was used to survey a sample of 120 participants (30 emergency medicine registrars and 90 prehospital emergency care practitioners: 30 Basic Life Support, 30 Intermediate Life Support, and 30 Advanced Life Support). An expert panel created the survey to ensure content validity and survey questions were designed to assess the perceived and actual knowledge of participants. We hypothesised that there would be significantly higher levels of perceived and actual knowledge in emergency medicine registrars compared to emergency care practitioners. An open-ended question on how participants felt dealing with child abuse was qualitatively analysed using thematic analysis.

Results: There were significant differences in the levels of perceived knowledge (58\% of emergency medicine registrars agreed that they felt adequately trained overall, versus $39 \%$ of emergency care practitioners; $-19 \%$ difference, $95 \% \mathrm{Cl}$ $-26 \%$ to $-12 \%)$ and actual knowledge (83\% of emergency medicine registrars with correct answers, versus $62 \%$ of emergency care practitioners; $-21 \%$ difference, $95 \% \mathrm{Cl}$ $-26 \%$ to $-16 \%$ ) among participants. Themes that emerged from qualitative analysis included personal distress, retaliation, frustration, medical system frustration, and personal competence concerns.

Discussion: Significant perceived and actual knowledge deficits of child abuse management exist among both emergency care practitioners and emergency medicine registrars in this setting. Future interventions should address the need for guidelines and increased training opportunities to ensure the health and safety of abused children.

Reproduced with permission 
emj.bmj.com

Official Journal of the Royal College of Emergency Medicine

Ultrasound-guided intravenous catheter survival impacted by amount of catheter residing in the vein

Ananda Vishnu Pandurangadu, Jared Tucker, Abigail R Brackney, Amit Bahl Published Online First: 18 July 2018. https://doi.org/10.1136/emermed-2017-206803

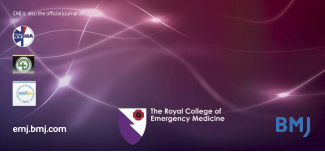

Objective: Ultrasound (US)-guided peripheral IVs have a high failure rate. We explore the relationship between the quantity of catheter residing within the vein and the functionality of the catheter over time.

Methods: This was a prospective, observational single-site study. Adult ED patients with US-guided IVs had the catheter visualised under ultrasound post-placement. IV placement time and catheter length residing in the vein was obtained. Exclusions included catheter not visualised, patient discharged from ED unless IV failed, <24 hour hospitalisation unless IV failed or patient self-removed IV.

Results: 113 patients were enrolled; 27 were excluded. Of the 86 study subjects, 29 (33.7\%) patients' IVs failed and 57
(66.3\%) remained functional. Median time to IV failure was 15.6 hours. $100 \%$ of IVs failed when $<30 \%$ of the catheter was in the vein; $32.4 \%$ of IVs failed when $30 \%-64 \%$ of the catheter was in the vein; no IVs failed when $\geq 65 \%$ of the catheter was in the vein $(p<0.0002)$. The HR was $0.71(95 \%$ $\mathrm{Cl} 0.60$ to 0.83 ), and for every $5 \%$ increase of catheter in vein, the hazard of the IV failing decreases by $29 \%(p<0.0001)$.

Conclusion: The quantity of catheter residing in the vein is a key predictor of long-term functionality of US-guided IVs and is strongly associated with the hazard of failure within 72 hours. Catheter failure is high when $<30 \%$ of the catheter resided in the vein. Optimum catheter survival occurs when $\geq 65 \%$ of the catheter is placed in the vein

\section{Emergencias}

emergencias.portalsemes.org/English

Official Journal of the Spanish Society of Emergency Medicine

Intubation through 2 supraglottic airway device in cervical spine immobilization: a randomized trial of residents' use of the intubating laryngeal mask airway and the intubating laryngeal tube in manikins

Dawid Aleksandrowicz, Tomasz Gaszyński

Cited: Aleksandrowicz D, Gaszyski T. Intubation through 2 supraglottic airway device in cervical spine immobilization: a randomized trial of residents' use of the intubating laryngeal mask airway and the intubating laryngeal tube in manikins. Emergencias. 2018;30:186-9.

http://emergencias. portalsemes.org/descargar/intubationthrough-2-supraglottic-airway-device-in-cervical-spine-immobiliza tion-a-randomized-trial-of-residents-use-of-the-intubatinglaryngeal-mask-airway-and-the-intubating-laryngeal-tube-inmanikins/english

Objective: The aim of this study was to compare the intubating laryngeal mask (iLM) airway and the new intubating laryngeal tube (iLTS-D) in use by residents with minimal previous intubation experience during simulated conditions of reduced cervical spine mobility.

Methods: Thirty first-year residents in anesthesiology participated in the study (18 women). All participants had minimal intubation experience (fewer than 10 previously performed intubations) and were novices in the specialty. Both devices were used by each participant after random assignment of order. We recorded the time required to insert the device and start to ventilate through it (T1) and the time from insertion and intubation to successful ventilation (T2). Efficacy of intubation and each resident's assessment of ease of use were also assessed.

Results: The residents' mean (SD) T1 values were similar for the 2 devices (iLMA, 15.3 [5.5] seconds; iLTS-D, 15.4 [5.5] seconds; $P=$.938). T2 was shorter with the iLTS-D (25.4 [8.6] seconds vs 31.9 [8.8] seconds with the iLMA; $P=.005)$. There were no failed intubation attempts with the iLTS-D.

Conclusions: The new iLTS-D may be a good alternative to the iLMA because a patient can be intubated and successfully ventilated in less time. The rate of successful intubation is also better with the iLTS-D.

Keywords: Supraglottic airway device. Intubation. Manikins. Cervical spine immobilization. Residents. 


\section{Hong Kong Journal of Emergency Medicine}

Hong Kong

Journal of

Emergency Medicine

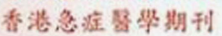

$\Leftrightarrow \rightarrow \infty$

Official Journal of the Hong Kong College of Emergency Medicine

Emergency physicians' attitudes to implementing ultrasound in Dutch emergency departments after a 2-day training: A qualitative Study

Rein Ketelaars, Esther Van Heumen, Lambert P Baken, Marja Witten, Gert Jan Scheffer, Yvonne Engels and Nico Hoogerwerf

Introduction: Diagnostic ultrasound is increasingly used by nonradiologists in trauma victims and critically ill patients. In the emergency department, the extended focused assessment with sonography for trauma and Polytrauma Rapid Echo-evaluation Program protocol are often used to assess these patients. Dutch Polytrauma Rapid Echo-evaluation Program-trained Emergency physicians are implementing the use of ultrasound in the emergency department but might encounter barriers to overcome. This study aims to explore individual experiences of Dutch emergency physicians.

Method: We performed a qualitative study by conducting semi-structured interviews in Dutch emergency physicians working in a Level 2 emergency department that completed the 2-day Polytrauma Rapid Echo-evaluation Program course at least 1 year before the interviews. Data were analyzed using directed content analysis.
Results: Eight emergency physicians employed by eight different hospitals were interviewed. Thirteen categories were identified in the transcribed interviews and these were combined into four general themes: (1) the desire to develop the Emergency Medicine specialty, both nationally and local; (2) incentives to start using ultrasound; (3) exploring practical applications of ultrasound; and (4) barriers faced while implementing emergency physician-performed ultrasound on the emergency department. The interviewees regard the course to be a solid base and are eager to independently perform ultrasound examinations, although challenges are faced.

Conclusion: This exploratory study provides essential insight in Dutch emergency physicians implementing ultrasound in their emergency department. It shows that there is a need to develop a quality assurance system and it identified barriers that have to be dealt with.

Keywords: Emergency Medicine, ultrasonography, education, qualitative research. 